\title{
Mid-gestational exposure to histone deacetylase inhibitor suberoylanilide hydroxamic acid influence cortical interneuron and astrocyte in mouse brain
}

\author{
Nunung Yuniarti ${ }^{1,2, *}$, Berry Juliandi ${ }^{1,3}$, Tsukasa Sanosaka ${ }^{1}$, and Kinichi Nakashima ${ }^{1}$ \\ ${ }^{1}$ Laboratory of Molecular Neuroscience, Graduate School of Biological Sciences, Nara Institute of Science and Technology, 8916-5 \\ Takayamacho, Ikoma, Nara, 630-0192 Japan \\ ${ }^{2}$ Laboratory of Pharmacology and Toxicology, Department of Pharmacology and Clinical Pharmacy, Faculty of Pharmacy, Universitas \\ Gadjah Mada, Sekip Utara, Sleman, Yogyakarta 55281, Indonesia \\ ${ }^{3}$ Division of Animal Biosystematics and Ecology, Department of Biology, Bogor Agricultural University, Jalan Raya Darmaga, Bogor 16680 , \\ Indonesia \\ ${ }^{*}$ Corresponding author: nunung@mail.ugm.ac.id
}

\begin{abstract}
Suberoylanilide hydroxamic acid (SAHA) has been reported preclinically to diffuse across the placenta and to be found in fetal plasma, suggesting that it can influence the fetus if taken by a pregnant cancer patient. In utero exposure of SAHA to mouse embryos during mid-gestation was found to perturb corticogenesis. However, the influence of in utero administration of SAHA to mouse embryos during mid-gestation on astrocyte, glial cell, and inhibitory neurons (interneurons) is yet to be reported. Pregnant dams were divided into control and SAHA groups and given methyl cellulose (as control) and SAHA orally once a day for 3 days during mid-gestation, starting from embryonic day (E)12 until E14. Astrocyte, interneuron, and behavior analyses were performed on the pups from postnatal day 7 until adulthood ( 3 months old). Brains were harvested and immunohistochemistry, Western Blot, and RT-PCR were performed on their cortex area. Transient exposure of SAHA to mouse embryos resulted in a decrease and increase in cortical astrocyte and interneuron, respectively. Meanwhile, adult SAHA mice displayed significantly increased anxiety, decreased memory, altered long-term cognitive functions, and reduced social interactions. Our study suggests that exposure to SAHA during prominent neurogenic periods might imbalance the normal excitatory:inhibitory neuron ratio required for the precise regulation of physiological functions in the brain.
\end{abstract}

KEYWORDS astrocyte; cortex; interneurons; neuronal cells; SAHA

\section{Introduction}

Stem cells drive the formation of tissues and organs through the development and remain in adult niche settings for tissue repair and maintenance. In early development, embryonic stem cells (ESCs) present in the inner cell mass of the blastocyst sense gradients of differentiation factors that instruct the formation of mesoderm, endoderm, and ectoderm. Specification of the central nervous system (CNS) begins with neural tube formation from ectoderm. ESCs develop into neural stem cells (NSCs). NSCs can self-renew and are multipotent, can give rise to its progenitors and in addition to neurons instead of glial cells (astrocytes, oligodendrocytes) in the CNS. Thus, owing to the self-renew ability and potential to generate various neural cell types, NSCs has profound implications for brain form and function as well as the repair of damaged tissue (e.g. spinal cord injury), neurodegeneration (Parkinson disease), and brain tumors (Ramasamy et al. 2013).
During corticogenesis where neurogenesis precedes gliogenesis, NSC undergoes fate restriction due to their potency change. In the neurogenic phase, NSCs produce different types of neurons populated in the different layer cortex. Neurogenesis occurred in an inside-out fashion where deep-layer (DL) neuron are arising and migrate first, while upper layer (UL) neuron are born and migrate later. DL neurogenesis occurs during mid-gestation, whereas UL neurogenesis occurs around late gestation followed by gliogenesis. The corticogenesis is complete on postnatal day 7 (Hevner et al. 2003).

Histone deacetylases (HDACs) are the chromatin modifiers that can epigenetically regulate NSC fate choice. Many works have identified that the inhibition of HDACs by histone deacetylase inhibitors (HDACIs) in vitro increased global histone acetylation and drove NSCs toward neuronal lineage over glial ones. Moreover, our group found the role of histone acetylation in the specification of UL neurons in late corticogenesis through HDACI treat- 
ment of mouse ESCs culture system that mimics corticogenesis in vitro (Namihira et al. 2008; Okano and Temple 2009; Juliandi et al. 2010a). In addition, we also reported that in utero exposure of SAHA to mouse embryos during mid-gestation, the time when prominent neurogenesis occurred, resulted in reduced NSC marker expression and perturbed corticogenesis by increased and decreased number of UL and DL cortical excitatory neurons, respectively. Furthermore, a determinant of UL neuronal lineage Satb2 was also up-regulated, whereas those of DL ones, Fezf2 and Ctip2, were down-regulated by SAHA treatment. UL neurogenesis was enhanced due to the increase of proliferating Tbr2+ intermediate progenitor cells that are committed to differentiation into UL neuron following SAHA administration. An identical effect was observed in vitro upon SAHA application to differentiating embryonic NSCs derived from mouse cortices. Collectively, these results suggest that proper regulation of HDACs is critical for precise embryonic corticogenesis (Yuniarti et al. 2013).

Suberoylanilide hydroxamic acid (SAHA) is one of the epi-drugs developed for cancer treatment that works epigenetically by inhibiting HDACs (O'Connor et al. 2006; Marks 2007). SAHA has been reported to diffuse across placenta and found in fetal plasma in preclinical studies, suggesting that it can influence fetuses if taken by pregnant cancer patients. The defect affected by fetal exposure to SAHA apparently persisted. In adult hippocampus of mice that treated by SAHA during mid-gestation, the number of NSC was still lower (Yuniarti et al. 2013). Given the fact that NSCs lining the embryonic neural tube give rise to the entire repertoire of neurons, astrocytes, and oligodendrocytes of the adult CNS, the decreased of NSCs in embryonic and adult SAHA-treated brains would influence the subsequent generation of glial cells, astrocytes and oligodendrocytes, that generally follows neurogenesis in the developing mammalian brain. However, there is no report about the influence of in utero administration of SAHA to mouse embryos during mid-gestation on astrocyte, one of the glial cells that support life of a nerve cell, and on inhibitory neurons (interneurons) considering that to support physiological function of brain particularly in cortical area required normal excitatory:inhibitory neuron ratio of 5:1.

\section{Materials and methods}

\subsection{Drug preparation}

Suberoylanilide hydroxamic acid (SAHA; Cayman Chemical, Ann Arbor, MI) was dissolved in dimethyl sulfoxide (DMSO; Nacalai Tesque, Kyoto, Japan) and then dose formulations were prepared as suspensions in $0.5 \%(\mathrm{w} / \mathrm{v})$ methyl cellulose (Wako, Osaka, Japan). The formulations were freshly prepared and briefly mixed before use.

\subsection{Animals treatment}

All mice used in this study were handled according to the animal experimentation guidelines of Nara Institute of Science and Technology that comply with National Institutes of Health Guide for the Care and Use of Laboratory Animals and animal treatment guidelines for preclinical study of Integrated Testing and Research Laboratory Universitas Gadjah Mada. All efforts were made to minimize the number of animals used and their suffering. Mice were individually housed on 12-h light/dark cycle at a constant temperature of $23-24^{\circ} \mathrm{C}, 50-70 \%$ humidity with free access to pellet diet and water. Time-pregnant C57BL/6J mice were orally administered with SAHA $(50 \mathrm{mg} / \mathrm{kg}$ ) or equal volume of $10 \%(\mathrm{v} / \mathrm{v})$ DMSO in methyl cellulose as Control, once a day for 3 consecutive days starting from embryonic day (E) 12.5 until E14.5, a period of prominent cortical neurogenesis. The pregnant mice in Control and SAHA groups were allowed to give birth and their offsprings were sacrificed after 7 days old (for astrocyte analysis). For interneuron analysis and behavior test, the same groups of 3 months old mice were used. Another set of pregnant mice in Control and SAHA groups were sacrificed on E15.5 for proliferating cell analysis. Brains were harvested and immunohistochemistry, Western Blot, and RT-PCR were performed to their cortex area.

\subsection{Tissue preparation}

Pregnant dams were sacrificed by cervical dislocation. Embryos were removed by cesarean section and immediately embryonic brains were collected in ice-cold phosphate buffered saline (PBS). Postnatal brains were collected after pups were deeply anesthetized by hypothermia before transcardially perfused with PBS and then $4 \%$ paraformaldehyde (PFA) in PBS. To harvest adult brains, mice were deeply anesthetized with i.p. injection of somnophentyl and perfused via the ascending aorta with PBS and PFA in PBS afterward. The dissected brains were fixed in 4\% PFA in PBS, followed by incubation in a series of sucrose gradient in PBS before being embedded in Optimal Cutting Temperature compound (Sakura Finetek, Torrence, CA) and stored at $-80^{\circ} \mathrm{C}$ until use. Cryostat sections of embryonic brains (in coronal plane with $20 \mu \mathrm{m}$ thickness), postnatal and adult brains (in coronal plane with $40 \mu \mathrm{m}$ thickness) were serially cut on a Leica CM 1900 (Leica Microsystems, Wetzlar, German) and mounted on MAS-coated glass slides (Matsunami Glass), while postnatal and adult brains were floated with PBS in 6-well or 12-well chamber slides (Nunc, Greiner). Before used for immunohistochemistry, the postnatal and adult brain sections were washed 3 times with PBS and then mounted on MAS-coated glass slides.

\subsection{Immunohistochemistry}

Brain sections were washed 3 times with PBS and then incubated in blocking solution (PBS containing 3\% FBS and $0.1 \%$ Triton $\mathrm{X}-100$ ) for $3 \mathrm{~h}$ at room temperature (RT). 
Next, sections were incubated overnight at $4^{\circ} \mathrm{C}$ with appropriate primary antibodies. The primary antibodies used were rabbit anti-Parvalbumin (1:500; Abcam) and mouse anti-pH3 (1:1000; Cell Signaling, Danvers, MA), and rabbit anti-cleaved Notch (1:100; Cell Signaling). Antigen retrieval was conducted by Antigen Retrieval solution (Dako, Glostrup, Denmark) for $15 \mathrm{~min}$ at $90^{\circ} \mathrm{C}$ in water bath for detection of some antigens when using Notch and Parvalbumin antibodies. After 3 washes with PBS, the sections were incubated for 1-2 h at RT with the appropriate secondary antibodies. The secondary antibodies used were Cy3-conjugated donkey anti-mouse and FITC-conjugated donkey anti-rabbit (all 1:500; Jackson ImmunoResearch Laboratories, West Grove, PA). After 3 washes with PBS, nuclei were stained for $15 \mathrm{~min}$ at RT with Hoechst 33258 (Nacalai Tesque). Sections were washed with PBS, cover slips were placed on the sections with Immu-Mount (Thermo Scientific, Pittsburgh, PA), and viewed and photographed using fluorescence microscope Axiovert 200M or Zeiss LSM 710 (Carl Zeiss, Gottingen, Germany) equipped with a camera and appropriate epifluorescence filters.

\subsection{Quantitative real-time PCR (qRT-PCR)}

Total RNAs from embryonic and postnatal cortices were isolated using Sepasol-RNA I Super G (Nacalai Tesque), and treated with DNase I (Promega, Madison, WI). cDNAs were synthesized from $1 \mu \mathrm{g}$ total RNA with SuperScript VILO cDNA Synthesis Kit (Invitrogen) as recommended by the manufacturer. qRT-PCR was performed by MX3000p (Agilent Technologies, Santa Clara, CA) using KAPA SYBR FAST qPCR Master Mix Universal kit with ROX as a reference dye (KAPA Biosystems, Boston, MA). The expression of target genes was normalized to that of glyceraldehyde 3-phosphate dehydrogenase (GAPDH). The gene-specific primers were as follows (5'3'): Hes5: Hes5-S, AAGAGCCTGCACCAGGACTA; Hes5-AS, CGCTGGAAGTGGTAAAGCA; NFIA: NFIA-S, GGCATACTTTGTACATGCAGC; NFIA-AS, CCTGATGTGACAAAGCTGTCC; NFIB: NFIB-S, TTTTTGGCATACTACGTGCAGG; NFIB-AS, TCTGATACATTGAAGACTCCG and GAPDH: GAPDH-S, ACCACAGTCCATGCC ATCAC; GAPDH-AS, TCCACCACCCTGTTGCTGTA.

\subsection{Cell quantification}

Coronal sections from at least 3 different areas of medial ganglion eminence (MGE) and lateral ganglion eminence (LGE) were carefully matched between groups based on Hoechst staining to examine $\mathrm{pH} 3+$ proliferating cells. Cortical area was selected for quantification of interneuron marker (Parvalbumin) expression in adult brain. The number of marker-positive cells was calculated manually by using a fluorescence microscope Axiovert 200M (Carl Zeiss) equipped with camera and appropriate epifluorescence filters within the indicated area. The percentage of marker- positive cells was counted among Hoechst+ cells within the indicated area.

\subsection{Western blot analysis}

Western blot analysis was performed as described previously (Kohyama et al. 2010). In brief, the postnatal and adult cortices were isolated, lysed, and the protein concentration in each sample was determined by the Bradford method. Twenty $\mu$ g protein samples of each total cell extract were separated by 5-20\% gradient using SDS polyacrylamide gels (e-PAGEL; ATTO Corp., Tokyo, Japan), transferred to a nitrocellulose membrane (GE Healthcare Life Sciences, Pittsburgh, PA), and probed with antigfap (1:1000, mouse IgG, Millipore), anti-Parvalbumin (1:500, rabbit, BioScience), anti-Somatostation (1:500, rabbit, Santa Cruz), or anti-GAPDH (mouse IgG, Millipore) antibodies. Signals were detected with anti-rabbit and anti-mouse IgG of horseradish peroxidase-conjugated secondary antibodies (Jackson Immunoresearch Lab.) using an ECL kit (GE Healthcare, Buckinghamshire, UK). Expression of gfap ( $50 \mathrm{kD}$ ), Parvalbumin ( $12 \mathrm{kD}$ ), and Somatostatin ( $17 \mathrm{kD}$ ) were detected. The amounts of proteins loaded in each slot were normalized to those of GAPDH ( $38 \mathrm{kD}$ ). The relative density of the protein expression was analyzed by ImageJ software $(\mathrm{NIH}$, Bethesda, MD).

\subsection{Behavioral test}

Experimental apparatuses and image analyzing software were obtained from O’Hara and Co., Ltd., Japan. Image analyzing software (Image OF4, Image LD2, Image EP2 and Image FZ2) was developed from the public domain ImageJ software. All experiments were done with 12 mice per group and were conducted between 13:30 and 16:30. The level of background noise during behavioral testing was about $50 \mathrm{~dB}$. After each trial, the apparatuses were wiped and cleaned.

\subsubsection{Open field test}

The locomotor activity was measured for $10 \mathrm{~min}$ using an open field apparatus made of white plastic (50 x 50 $\mathrm{x} 40(\mathrm{H}) \mathrm{cm})$. An LED light system was positioned 50 $\mathrm{cm}$ above the center of the field (50 lux at the center of the field). Total distance traveled (in $\mathrm{cm}$ ), time spent in the central area (30\% of the field) (in second), and the frequencies of movement were measured. Mice were put on a large, open, bright, and white area for $5 \mathrm{~min}$ and the behaviors were observed afterward. The observed parameter were line crossing, center square entries, center square duration, rearing, stretch attend posture, grooming, and freezing.

\subsubsection{Social interaction test}

The apparatus used for social interaction test consisted of one transparent chamber divided into three compartments. The three-chamber test assessed cognition in the form of 


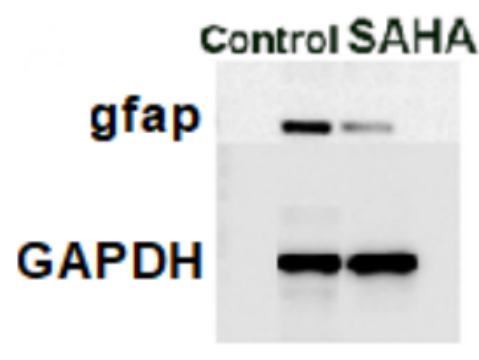

(a)

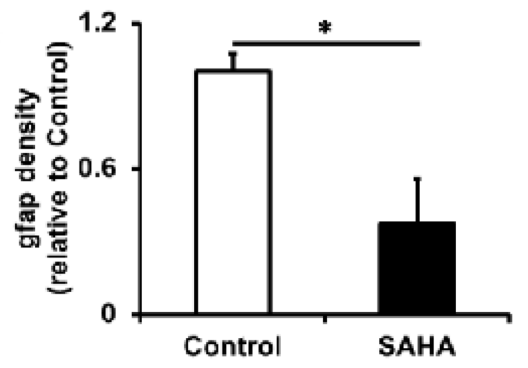

(b)

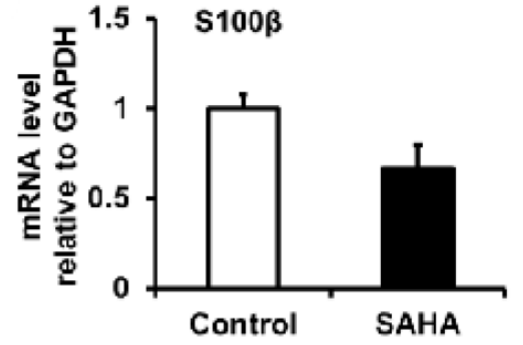

(c)

FIGURE 1 Mid-gestational HDAC inhibition by SAHA led to the suppression of astrocyte differentiation in P7 cortices. Western blot (a) and its quantification (b) showed lower expression of gfap protein in SAHA-treated neonatal cortices. ${ }^{*} p<0.05 ;$ Student's t-test; $n=3$. RT-PCR of astrocytic gene $S 100 \beta$ supported this result $(c)(n=3)$.

general sociability and interest in social novelty in mouse models of CNS disorders. Mice normally preferred to spend more time with another mouse (sociability) and investigated a novel intruder more so than a familiar one (social novelty). Based on these inclinations, the threechamber test could help identify rodents with deficits in sociability and/or social novelty.

Testing occurred in three sessions within a threechambered box with openings between the chambers. After habituation to the empty box, the mouse encountered a never-before-met intruder under one wire cup and an empty wire cup in the "sociability" session. The mouse then encountered the first intruder as well as a second never-before-met intruder under another wire cup in the "social novelty" session. Observation of the mice behavior was performed for $10 \mathrm{~min}$. The observed parameters were duration and frequency of contact/interaction between tested mouse and intruder 1 mouse and duration and frequency of contact/interaction between tested mouse and intruder 1 and 2 mice.

\subsubsection{Passive avoidance test}

The apparatus used for the light/dark transition test consisted of a cage $(21 \times 42 \times 25(\mathrm{H}) \mathrm{cm})$ divided into two compartments by a partition with an opening. One compartment was brightly illuminated (250 lux), whereas the other compartment was dark (2 lux). When a mouse was placed into the dark area, it was trained with electrical shock. The mice were trained to avoid a 'punishment': electrical shock (Vohora et al. 2000). Mice were put in bright compartment for $10 \mathrm{~s}$ and the partition was opened. The mice will move to the dark compartment and here the electrical shock was given to them. Mice were rested for $20 \mathrm{~min}$ and the above method was performed again. Observed parameter were latency time 1 (short term memory; the duration of the mouse in moving to the dark compartment from the bright one (for the first time)) and latency time 2 (long term memory; the duration of the mouse in moving to the dark compartment from the bright one after resting time for $20 \mathrm{~min}$ (for the second time)).

\subsection{Statistical analysis}

Statistical analyses were performed from at least 3 pups or mice of each group. Statistical comparisons were made by Student's t-test (for 2-groups comparison) and ANOVA (for multiple groups comparison) with Tukey post hoc tests.

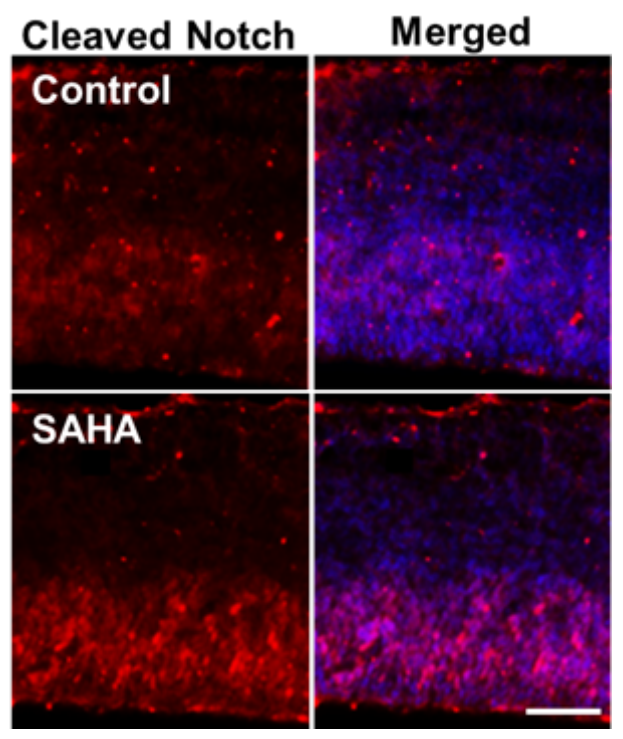

(a)

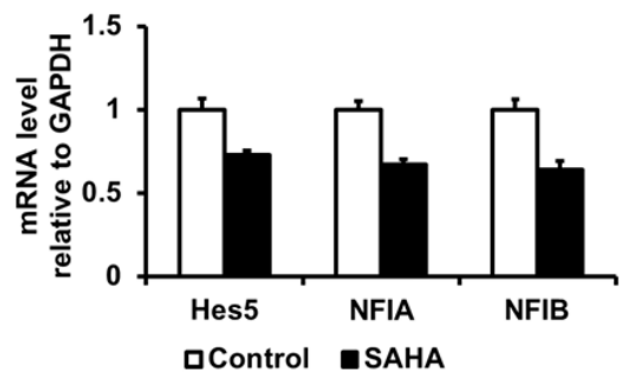

(b)

FIGURE 2 Antigenically recognizable Notch ligand NICD was increased by SAHA treatment (red) (a) probably owing to the increased of IPC number in embryonic cortex. The expression of Notch signaling pathway downstream molecules including Hes5, NFIA, and NFIB were reduced by SAHA administration (b) $(n=3)$. 


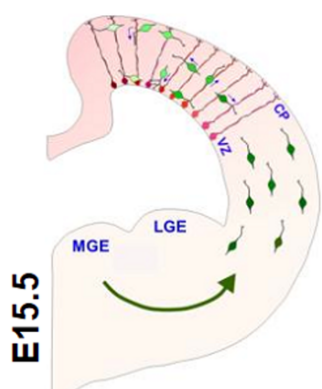

(a)

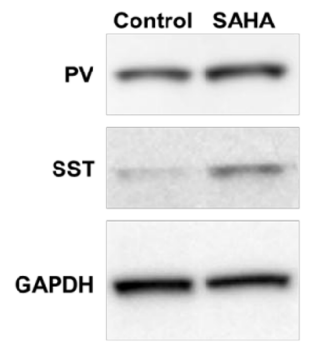

(d)

\section{E15.5}

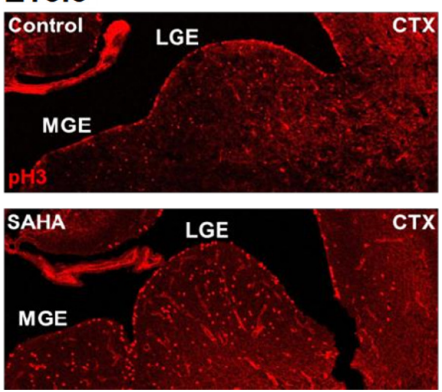

(b)
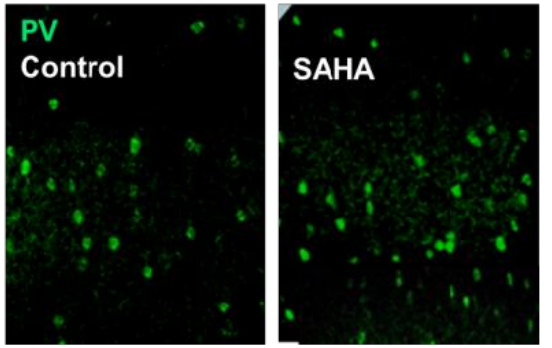

(e)

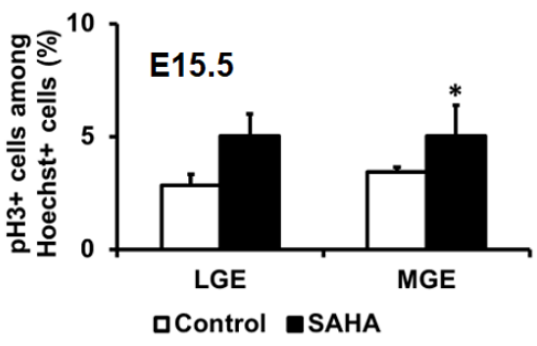

(c)

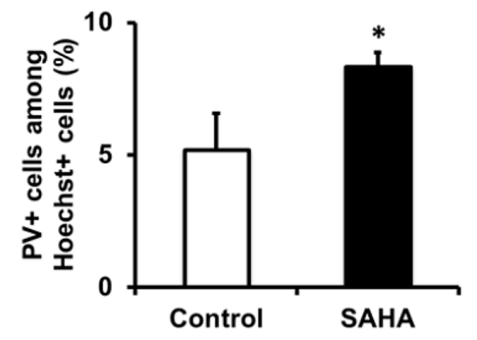

(f)

FIGURE 3 SAHA increased cortical interneuron in adult brains perhaps due to increased number of proliferating cells within the embryonic LGE and MGE. (a) Interneurons (green) originate from NSC located outside the embryonic cortex, in the LGE and MGE of ventral telencephalon. Interneurons migrating into the cerebral wall from the MGE and LGE interact with NSC (red) and can exhibit changes in direction of migration after contacting NSCs. Interneurons can use glia fiber of NSCs as a scaffold upon which to migrate as they ascend to the cortical plate (CP) or descend in the direction of the ventricular zone (VZ). Once the interneurons invade the cortex from the MGE and LGE, differential interactions between interneurons and NSC scaffold and localized multidirectional migration of interneurons influenced by local guidance cues may facilitate interneuronal positioning within distinct domains of the developing cerebral cortex. Blue arrows indicate direction of migration. (b-c) Immunostaining (b) and the number of cells (c) that are proliferative (pH3+) during embryonic development in the LGE and MGE increased following SAHA application. (d-f) SAHA-treated adult cortices showed increased Parvalbumin- and Somatostatin-expressing interneurons relative to Control ones. Ctx: Cortex.

\section{Results and discussion}

\subsection{SAHA reduces cortical astrocytogenesis}

Considering the fact that NSCs lining the embryonic neural tube give rise to the entire repertoire of neurons, astrocytes, and oligodendrocytes of the adult CNS, we hypothesized that the decrease of NSCs in embryonic and adult SAHA-treated brains would influence the subsequent generation of glial cells, astrocytes and oligodendrocytes that generally follows neurogenesis in the developing mammalian brain. To determine the effect of in utero HDAC inhibition by SAHA on gliogenesis particularly astrocytogenesis, we quantified astrocyte markers, gfap and S100 $\beta$, expression of postnatal SAHA cortices using western blot and RT-PCR, respectively. Immunoblotting analysis and relative band density of gfap protein in SAHA-treated neocortex is reduced until 0.4 compared with the Control (Figure $1 \mathrm{a}-\mathrm{b})$. mRNA level of $\mathrm{S} 100 \beta$ astrocytic gene confirmed this phenotype (Figure 1c). Collectively, this result indicates that SAHA repressed the production of cortical astrocyte. We assume that this phenotype is the consequence of the reduction of NSCs thus lead to the reduced number of astrocyte progenitors in SAHA-treated cortices.
Other possibility related to Notch signaling is the reduction of NFIA expression by SAHA administration may cause decreased astrocytic differentiation potency of midgestational NSC (Figure 2) since it has been suggested that NFIA, which bind to astrocytic gene promoters and act as downstream of the Notch signaling pathway to potentiate astrocytic differentiation of mid-gestational NSCs, accelerated demethylation of astrocytic gene promoters by preventing DNMT1 from binding to them and thus allowed precocious astrocytic differentiation (Namihira et al. 2009; Juliandi et al. 2010b).

\subsection{SAHA increased cortical interneuron generation}

A prominent trait of the mammalian cerebral cortex is its complex cellular architecture, which relies on the development of a diversity of neurons extensively interconnected into functional networks. Cortical excitation and inhibition are executed by highly heterogeneous populations of glutamatergic excitatory neurons and GABAergic inhibitory interneurons, respectively. The establishment of correct reciprocal positioning and interactions between these two broad neuronal classes is very important for balanced electrical activity and normal cortical func- 
TABLE 1 SAHA increased frequency of stretch attends posture, grooming, freezing and decreased line crossing and center square duration in open field test. Data represented in mean $\pm S D(n=5)$.

\begin{tabular}{llll}
\hline Groups and Parameters & Normal control & $\begin{array}{l}\text { Ethanol-induced brain disorder } \\
\text { control }\end{array}$ & SAHA \\
\hline Stretch attend posture & $6.2 \pm 1.6$ & $15.6 \pm 6.0$ & $13.4 \pm 3.1^{*}$ \\
Line crossing & $77.8 \pm 27.3$ & $56.4 \pm 20.3$ & $49.7 \pm 19.7^{*}$ \\
Grooming & $30.6 \pm 12.2$ & $76.2 \pm 26.0$ & $65.6 \pm 17.4^{*}$ \\
Freezing & $4.2 \pm 4.6$ & $41.2 \pm 11.9$ & $19.0 \pm 8.5^{*}$ \\
Center square duration & $13.4 \pm 10.5$ & $3.0 \pm 3.3$ & $2.4 \pm 3.1^{*}$ \\
Center square entries & $3.0 \pm 2.5$ & $0.8 \pm 0.8$ & $2.0 \pm 2.6$ \\
Rearing & $11.6 \pm 6.8$ & $6.8 \pm 5$. & $16.8 \pm 13.9$ \\
\hline
\end{tabular}

$* p<0.05$ significantly different relative to control.

TABLE 2 SAHA changed social affiliation affect: reduced interaction within the groups in social interaction test. Data represented in mean $\pm S D(n=5)$.

\begin{tabular}{lll}
\hline $\begin{array}{l}\text { Frequency and } \\
\text { groups }\end{array}$ & $\begin{array}{l}\text { Frequency contact } \\
\text { of mice with } \\
\text { empty wire cup }\end{array}$ & $\begin{array}{l}\text { Frequency } \\
\text { contact of mice } \\
\text { with intruder } 1\end{array}$ \\
\hline $\begin{array}{l}\text { Normal control } \\
\text { Ethanol-induced }\end{array}$ & $\begin{array}{l}4.1 \pm 3.7 \\
\text { brain disorder control }\end{array}$ & $14.5 \pm 13.4$ \\
SAHA & $6.9 \pm 2.5$ & $13.4 \pm 8.0$ \\
\hline
\end{tabular}

$* p<0.05$ significantly different relative to control.

TABLE 3 SAHA changed social novelty: frequency contact of SAHA mice to intruder mice was less than normal control in social interaction test. Data represented in mean $\pm S D(n=5)$.

\begin{tabular}{lll}
\hline $\begin{array}{l}\text { Frequency and } \\
\text { groups }\end{array}$ & $\begin{array}{l}\text { Frequency contact } \\
\text { of mice with } \\
\text { intruder } 1\end{array}$ & $\begin{array}{l}\text { Frequency } \\
\text { contact of mice } \\
\text { with intruder } 2\end{array}$ \\
\hline $\begin{array}{l}\text { Normal control } \\
\begin{array}{l}\text { Ethanol-induced } \\
\text { brain disorder control }\end{array}\end{array}$ & $13.3 \pm 5.7$ & $11.2 \pm 4.4$ \\
SAHA & $9.7 \pm 2.0$ & $7.5 \pm 1.6$ \\
\hline
\end{tabular}

tion including cognition, sensory perception, and motor function. Thus, the constant fraction of cortical excitatory:inhibitory (E:I) neurons circuit balance in 5:1 from near the start of cortical neurogenesis to adulthood (Sahara et al. 2012) is necessary. The disruption of E:I circuit balance due to reduced expression of cortical GABAergic neurons such as GAD67, Reelin, Somatostatin, Parvalbumin in the hippocampus, basal ganglia, or upper to layer 5 cortical layers of psychotic patients including schizophrenia (SZ), autism spectrum disorder (ASD), and bipolar disorder patients markedly reduces the effectiveness of the GABAergic inhibitory transmission that impinges on the dendrite and on the initial axon segments of excitatory neurons (Grayson et al. 2011). This deficit of inhibitory neurotransmitter disrupts the intermittent synchronization of excitatory neuron firing that is critical for normal neuronal function (Gonzalez-Burgos and Lewis 2008).
Our previous finding about the perturbation of postnatal cortical lamination due to the increased and decreased production of excitatory neuron in UL and DL neocortex, respectively (Yuniarti et al. 2013), following midgestational SAHA exposure might cause the inconstant E/I fraction thus lead to the alteration of excitatory neurons' firing and induce the abnormality of neuronal function in the cortex.

During development, excitatory neurons and interneurons are born in separate locations. The excitatory neurons are generated from NSC in the ventricular zone (VZ) and subventricular zone (SVZ) of embryonic cortex and typically migrate radially in an 'inside-out' sequence to form layers in the cortical plate (CP). Most, if not all, interneurons, however, are born from NSC located outside the cortex, in the lateral- and medial-ganglionic eminences (LGE and MGE) (Figure 3a) in ventral telencephalon, and migrate first tangentially from their birthplace to the proper cortical region, and then radially through the CP to reach their final laminar location. It seems that the LGE and MGE of the subcortical telencephalon also contribute cells to the neocortex and they are the primary sources of cortical interneurons in rodents (Anderson et al. 2002) including Parvalbumin- and Somatostatin-expressing interneurons (Xu et al. 2004). MGE NSC give rise to early born and Parvalbumin- and Somatostatin-expressing interneurons, which distribute at higher densities in deep cortical layers (Butt et al. 2005; Fogarty et al. 2007).

Because the generation of Parvalbumin and Somatostatin interneurons from NSC start emerged around E1214 in the MGE (Butt et al. 2005) and this is the time window when SAHA exposed to the pregnant dams, we first examined the cells in the MGE and LGE. Here we demonstrated that SAHA-treated mice have greater numbers of proliferating cells in embryonic LGE and MGE than Control ones (Figure 3b-c). Parvalbumin and Somatostatin interneurons can later be found throughout the brain, including hippocampus, thalamus, and cortex (Butt et al. 2005). Thus next, we investigated the Parvalbumin and Somatostatin expression within the adult cortex. Here we found that SAHA treatment led to the in- 
TABLE 4 SAHA changed short- and long-term memory by reducing latency time 1 and 2 in passive avoidance test. Data represented in mean $\pm S D(n=5)$.

\begin{tabular}{llllll}
\hline Latency time (second) & Groups & \multicolumn{3}{c}{ Day } \\
& & 1 & 2 & 3 & 4 \\
\hline Latency time 1 & Normal control & $103.0 \pm 66.3$ & $126.2 \pm 62.0$ & $170.4 \pm 15.7$ & $75.0 \pm 19.3$ \\
& Ethanol-induced brain disorder control & $25.4 \pm 20.7$ & $17.8 \pm 10.9$ & $88.8 \pm 69.0$ & $106.0 \pm 84.4$ \\
& SAHA & $55.4 \pm 17.3^{*}$ & $37.4 \pm 12.4 *$ & $75.0 \pm 24.8^{*}$ & $88.8 \pm 14.7 *$ \\
Latency time 2 & Normal control & $121.2 \pm 66.6$ & $148.2 \pm 49.6$ & $169.4 \pm 23.7$ & $125.4 \pm 59.8$ \\
& Ethanol-induced brain disorder control & $136.2 \pm 20.9$ & $148.0 \pm 51.0$ & $110.6 \pm 50.3$ & $63.2 \pm 69.9$ \\
& SAHA & $54.8 \pm 42.3$ & $99.6 \pm 54.6$ & $101.6 \pm 66.6$ & $45.0 \pm 33.0$ \\
\hline
\end{tabular}

$* p<0.05$ significantly different relative to control.

creased percentage of cortical Parvalbumin+ cells (Figure $3 e-f)$. Immunoblotting supported the increased expression of Parvalbumin- and Somatostatin-expressing interneurons in SAHA-treated adult cortices (Figure 3d).

Overall, the finding about the perturbation of postnatal cortical lamination due to the increased and decreased production of excitatory neuron in UL and DL neocortex, respectively (Yuniarti et al. 2013), along with the increased number of cortical inhibitory interneurons following midgestational SAHA application might deregulate the normal ratio between excitatory and inhibitory neurons required for the precise regulation of physiological functions in the brain. To investigate the functional brain in SAHA mice after they reach adulthood, we performed behavior test that related to cortical functions in the regulation of anxiety, learning and memory, and social interaction such as open field test, passive avoidance test, and social interaction test (Seo et al. 2013). SAHA mice at dose $50 \mathrm{mg} / \mathrm{kg}$ BW showed significantly increased depression, as shown in the increase in stretch attend posture, grooming and freezing and decreased line crossing and center square duration in the open field test, decreased short term memory and changed long term memory in the passive avoidance test, and reduction in social interaction by reducing the capability of mice to interact within their groups (Table 1-4).

The increased number of interneurons in the embryos exposed to HDACI SAHA in this study is in agreement with Grayson et al. (2011) by which treatment of neuronal progenitor cultured cells with various HDACIs (MS-275, VPA, TSA) led to a robust induction of interneuron mRNAs such as reelin and GAD67 as well as DNMT inhibitors. DNMT and HDAC inhibitors target DNMT1 and HDAC1 and facilitate the dissociation of DNMTcontaining repressor complexes from interneuron (as proposed for the reelin and GAD67 genes) promoters led to DNA demethylation, histone acetylation, and a relaxation of chromatin allows the recruitment of specific transcription factors, and the general transcriptional machinery to the promoters.

Collectively, these results suggest that change in epigenetic status that is histone acetylation of non-cancerous cells by pharmacological inhibition of HDACs following anti-cancerous SAHA exposure during mid-pregnancy could alter fetal and adult brain structure. These support the precaution of SAHA as anticancer agent for pregnant patient by FDA, that is pregnancy category $\mathrm{D}$ meaning that SAHA has potential hazard to the fetus. All in all, these findings might contribute to the pharmaceutical therapy guideline not only for cancer treatment but also for treatment of other diseases employing HDACIs. Regarding that SAHA has a potential hazard to the fetus, it should not be prescribed to pregnant patient or to patient who planned to become pregnant while taking this drug.

\section{Conclusions}

The consequences of prenatal SAHA exposure in adulthood are suppressed cortical astrocyte by downregulating NFIA expression thus decreased the potency of astrocyte differentiation, and increased cortical interneuron by increasing number of proliferating cells that might be NSC committed to PV and SST interneurons. This defect may alter the composition of cells within SAHA-treated cortex thus impact on brain function since SAHA mice at dose $50 \mathrm{mg} / \mathrm{kg}$ BW showed significantly increased anxiety, decreased memory and long-term cognitive functions, and reduced social interaction.

\section{Acknowledgments}

This work was supported by Ministry of Education, Culture, Sports, Science and Technology, Japan through NAIST Global COE Program (Frontier Biosciences: Strategies for survival and adaptation in a changing global environment) and Grant-in-Aid for Scientific Research on Innovative Area: Neural Diversity and Neocortical Organization, and by the Government of the Republik Indonesia through the Hibah Kompetensi 2016 Research Grant.

\section{Authors' contributions}

KN conceived the idea and developed the theoretical framework. NY carried out the experiments and wrote the manuscript in consultation with $\mathrm{BJ}$ and $\mathrm{KN}$. TS con- 
tributed to sample analysis. BJ and $\mathrm{KN}$ supervised the work.

\section{Competing interests}

The authors declare that they have no significant competing financial, professional or personal interests that might have influenced the performance or presentation of the work described in this manuscript.

\section{References}

Anderson SA, Kaznowski CE, Horn C, Rubenstein JLR, McConnell SK. 2002. Distinct origins of neocortical projection neurons and interneurons in vivo. Cereb Cortex 12(7):702-709. doi:10.1093/cercor/12.7.702.

Butt SJB, Fuccillo M, Nery S, Noctor S, Kriegstein A, Corbin JG, Fishell G. 2005. The temporal and spatial origins of cortical interneurons predict their physiological subtype. Neuron 48(4):591-604. doi:10.1016/j.neuron.2005.09.034.

Fogarty M, Grist M, Gelman D, Marín O, Pachnis V, Kessaris N. 2007. Spatial genetic patterning of the embryonic neuroepithelium generates GABAergic interneuron diversity in the adult cortex. J Neurosci. 27(41):10935-10946. doi:10.1523/JNEUROSCI.1629-07.2007.

Gonzalez-Burgos G, Lewis DA. 2008. GABA neurons and the mechanisms of network oscillations: implications for understanding cortical dysfunction in schizophrenia. Schizophr Bull. 34(5):944-961. doi:10.1093/schbul/sbn070.

Grayson D, Kundakovic M, Chen Y, Dong E, Guidotti A. 2011. Epigenetic regulation of GABAergic targets in psychiatry. In: A Petronis, J Mill, editors. Brain, behavior and epigenetics. Berlin: Springer Science \& Business Media. p. 23-40. doi:10.1007/978-3-64217426-1_2.

Hevner RF, Daza RAM, Rubenstein JLR, Stunnenberg H, Olavarria JF, Englund C. 2003. Beyond laminar fate: toward a molecular classification of cortical projection/pyramidal neurons. Dev Neurosci. 25(2-4):139151. doi:10.1159/000072263.

Juliandi B, Abematsu M, Nakashima K. 2010a. Chromatin remodeling in neural stem cell differentiation. Curr Opin Neurobiol. 20(4):408-415. doi:10.1016/j.conb.2010.04.001.

Juliandi B, Abematsu M, Nakashima K. 2010b. Epigenetic regulation in neural stem cell differentiation. Dev Growth Differ. 52(6):493-504. doi:10.1111/j.1440169X.2010.01175.x.

Kohyama J, Sanosaka T, Tokunaga A, Takatsuka E, Tsujimura K, Okano H, Nakashima K. 2010. BMPinduced REST regulates the establishment and maintenance of astrocytic identity. J Cell Biol. 189(1):159170. doi:10.1083/jcb.200908048.
Marks PA. 2007. Discovery and development of SAHA as an anticancer agent. Oncogene 26(9):1351-1356. doi:10.1038/sj.onc.1210204.

Namihira M, Kohyama J, Abematsu M, Nakashima K. 2008. Epigenetic mechanisms regulating fate specification of neural stem cells. Philos Trans R Soc Lond B Biol Sci. 363(1500):2099-2109. doi:10.1098/rstb.2008.2262.

Namihira M, Kohyama J, Semi K, Sanosaka T, Deneen B, Taga T, Nakashima K. 2009. Committed neuronal precursors confer astrocytic potential on residual neural precursor cells. Dev Cell 16(2):245-255. doi:10.1016/j.devcel.2008.12.014.

O’Connor OA, Heaney ML, Schwartz L, Richardson S, Willim R, MacGregor-Cortelli B, Curly T, Moskowitz C, Portlock C, Horwitz S, Zelenetz AD, Frankel S, Richon V, Marks P, Kelly WK. 2006. Clinical experience with intravenous and oral formulations of the novel histone deacetylase inhibitor suberoylanilide hydroxamic acid in patients with advanced hematologic malignancies. J Clin Oncol. 24(1):166-173. doi:10.1200/JCO.2005.01.9679.

Okano H, Temple S. 2009. Cell types to order: temporal specification of CNS stem cells. Curr Opin Neurobiol. 19(2):112-119. doi:10.1016/j.conb.2009.04.003.

Ramasamy S, Narayanan G, Sankaran S, Yu YH, Ahmed S. 2013. Neural stem cell survival factors. Arch Biochem Biophys. 534(1-2):71-87. doi:10.1016/j.abb.2013.02.004.

Sahara S, Yanagawa Y, O’Leary DDM, Stevens CF. 2012. The fraction of cortical GABAergic neurons is constant from near the start of cortical neurogenesis to adulthood. J Neurosci. 32(14):4755-4761. doi:10.1523/JNEUROSCI.6412-11.2012.

Seo TB, Cho HS, Shin MS, Kim CJ, Ji ES, Baek SS. 2013. Treadmill exercise improves behavioral outcomes and spatial learning memory through up-regulation of reelin signaling pathway in autistic rats. J Exerc Rehabil. 9(2):220-229. doi:10.12965/jer.130003.

Vohora D, Pal SN, Pillai KK. 2000. Effect of locomotor activity on the passive avoidance test for the evaluation of cognitive function. Indian J Pharmacol. 32(3):242245.

Xu Q, Cobos I, Cruz EDL, Rubenstein JL, Anderson SA. 2004. Origins of cortical interneuron subtypes. J Neurosci. 24(11):2612-2622. doi:10.1523/JNEUROSCI.5667-03.2004.

Yuniarti N, Juliandi B, MuhChyi C, Noguchi H, Sanosaka T, Nakashima K. 2013. Prenatal exposure to suberoylanilide hydroxamic acid perturbs corticogenesis. Neurosci Res. 77(1-2):42-49. doi:10.1016/j.neures.2013.06.004. 